\title{
Innovación y Productividad Manufacturera
}

\section{Innovation and Manufacturing Productivity}

\author{
Pedro Sánchez-Sellero', Mª Carmen Sánchez-Sellero², Francisco Javier Sánchez-Sellero³, \\ María Montserrat Cruz-González ${ }^{4}$
}

\begin{abstract}
Analizamos el efecto de la innovación sobre la productividad en la empresa manufacturera española. Así identificamos y justificamos los siguientes factores de innovación que determinan la productividad: actividades de investigación y desarrollo (I+D), gastos I+D, patentes, innovaciones de producto, innovaciones de proceso, organización interna de la innovación, externalización de las actividades de I+D, financiación pública de los gastos de I+D y colaboración exterior de la innovación. Las conclusiones de este trabajo basado en un análisis de regresión múltiple para el año 2010 a nivel de empresa, demuestran que las innovaciones de proceso y las actividades de I+D (especialmente en actividades internalizadas) están en mejores condiciones de favorecer la productividad de la empresa manufacturera española.
\end{abstract}

Keywords: innovación; productividad; industria y españa.

We analyze the effect of innovation on productivity in Spanish manufacturing firm. So, we identify and explain this effect of the following innovation factors on productivity: research and development (R\&D) activities and expenditures, patents, innovations in products and in process, internal organization of innovation, outsourcing R\&D activities, public funding in R\&D expenditures and external relationships of innovation. Our results are based on a multiple regression analysis in 2010 at the firm level. So, we show that innovations in process and R\&D activities (specially internal R\&D activities) are in a better condition to favor the productivity of Spanish manufacturing firms.

Keywords: innovation; productivity; industry and spain.

\footnotetext{
' Department of Business Administration, Universidad de Zaragoza, School of Engineering, Campus Río Ebro, 500I8, Zaragoza, Spain. Phone: 34976761000; e-mail: pedross@unizar.es

2 Department of Applied Economics, Universidad de La Coruña, Faculty of Economics and Business Administration, Campus Elviña, I507I, La Coruña, Spain. Phone: 3498I I67000; e-mail: c.sanchez@udc.es

3,4 Department of Business Administration, Universidad de Vigo, Faculty of Economics and Business Administration, Campus Lagoas-Marcosende, 363 IOVigo, Pontevedra, Spain.Phone: 349868I2489; e-mail: ${ }^{3}$ javiss@uvigo.es, ${ }^{4}$ mcruz@uvigo.es
}

ISSN: 07 I8-2724. (http://www.jotmi.org)

Journal of Technology Management \& Innovation (c) Universidad Alberto Hurtado, Facultad de Economía y Negocios. 


\section{Introduction}

La innovación favorece el desarrollo de la empresa, de la industria y del país en el que se lleva a cabo y es muy relevante para lograr una mayor productividad. Los factores mano de obra y capital han sido siempre claves en esta materia pero han dado paso a otros nuevos relacionados con la innovación.

En este trabajo se trata de encontrar los factores de innovación determinantes de la productividad, tales como actividades de $I+D$, gastos en $I+D$, número de patentes, innovaciones de producto, innovaciones de proceso, organización interna de la innovación, actividades I+D: internalización y externalización, financiación pública de la $I+D$, colaboración exterior de la innovación. Para ello, analizaremos el modo en que repercute cada factor de innovación y en qué sentido sobre la productividad de la empresa que lo lleva a cabo.

La estructura del trabajo es la siguiente: en primer lugar se analizan los factores determinantes de la productividad, y se proponen hipótesis para cada uno de ellos; en segundo lugar se presenta el modelo, los datos y la metodología que vamos a aplicar; en tercer lugar se centra el análisis de los resultados y discusión de los mismos; por último, se establecen las conclusiones.

\section{Factores de innovación determinantes de la produc- tividad}

El estudio del tópico de la innovación continua siendo un gran objeto de interés por lo que existen muchos estudios sobre la influencia de la innovación sobre la productividad. Así, algunos se centran sólo en el análisis de uno de ellos, un ejemplo son los trabajos de Benito (200I), Doraszelski y Jaumandreu (2007), Fluviá (1990), Grandón y Rodríguez Romero (1991) o López Pueyo y Sanaú Villarroya (1998).Todos ellos muy interesantes pero partiendo de la función de producción estudian la producción con respecto al capital I+D. Existen también otros estudios que guardan mayor similitud con el que aquí se plantea, donde los autores analizan el efecto simultaneo de varios factores sobre la productividad. Crépon y otros (1998) analiza el número de patentes y el gasto en I+D, Gu y Tang (2003) la inversión en I+D, patentes, inversión en maquinaria y equipos así como la organización interna y Rosell-Martínez y Sánchez-Sellero (20l I) estudian el efecto de la I+D y la inversión extranjera directa. Este trabajo pretende ser ambicioso y realizar un análisis más completo, no sólo centrarse en un único factor determinante. Pretende abarcar al igual que estos últimos autores, todos aquellos factores más destacados por la literatura como determinantes de la productividad. Para ello, a continuación se realiza un análisis detallado de la bibliografía, escogiendo los factores a analizar. Finalmente, aportamos sobre cada uno de ellos las citas que nos permiten formular las hipótesis (tabla I) que probamos en el análisis empírico. Actividades de I+D

Un gran número de autores han demostrado la importancia que tiene este factor con el aumento de la productividad.Ya Griliches (1979) estableció el modelo de stock de capital en I+D que analizaba la relación entre la inversión en I+D para lograr innovaciones y el crecimiento de la productividad. Pero no ha sido el único, Griffith y otros (2000) evidencian la importancia de las actividades I+D como factor determinante del crecimiento de la productividad en un grupo de industrias de doce países de la OCDE. La I+D estimula directamente el crecimiento mediante la innovación y también indirectamente a través de la transferencia de tecnología.Así, juega un papel fundamental en la convergencia de los niveles de productividad total de las industrias.

Otros estudios empíricos aplicados en un marco de la función de producción a la cual se le ha añadido como factor adicional las actividades I+D (Czarnitzki y Niall, 2007), han demostrado que éstas contribuyen a aumentar la productividad, la competitividad y por lo tanto el crecimiento a largo plazo y las perspectivas de empleo de la economía. Al igual que Barges-Gil y López (20II) los que tras estudiar el factor de I+D afirman que constituye la principal fuente de innovación y es uno de los determinantes de los incrementos de productividad de las empresas.

\section{Gastos de I+D}

El Observatorio de la Sostenibilidad en España realiza estudios anuales sobre la inversión en I+D en relación al PIB. En su estudio del año $201 \mathrm{I}$ concluyen que el gasto en $I+D$ de un país es un aspecto clave para incrementar la productividad y generar crecimiento a largo plazo, se considera a su vez un indicador fundamental del potencial económico de un país. Asimismo considera el gasto de España insuficiente en este tipo de partidas y más aún desde la situación de crisis y recortes a la que se está viendo sometida estos últimos años. Sin embargo el estudio de este factor se remonta a años atrás, ya Griliches y Mairesse (1984) estudiaron a 133 empresas de EE.UU. entre los años 1966 y 1977 llegando a la conclusión de que la productividad que éstas obtenían tenía una fuerte relación con el nivel de inversión en I+D que realizaban. Otros autores (Maté García y Rodríguez Fernández, 2002; Gu y Tang, 2003) dan la misma importancia a este factor, relacionando el gasto en $I+D$ con el crecimiento de la productividad.

El estudio que Maté García y Rodríguez Fernández (2002) realizan aplicando un modelo teórico que relaciona el crecimiento de la productividad del trabajo con el gasto en I+D de empresas españolas (base de datos de la ESEE para el 
período 1993-1999) concluye que la inversión en I+D por parte de las empresas tienen un efecto positivo y estadísticamente significativo en el incremento de la productividad. Tal y como ocurría con el factor actividades de I+D estos autores también encuentran importantes debilidades de inversión I+D en las empresas españolas, esta carencia puede suponer sobre ellas una pérdida de competitividad sobre otros países europeos los cuales han realizado un mayor esfuerzo sobre esta materia.

\section{Patentes}

Tal y como han estudiado algunos autores el número de patentes puede tener cierta influencia sobre la productividad obtenida. El trabajo de Crèpon y otros (1998) muestra la influencia que tiene la variable número de patentes realizadas como variable adicional en una función de producción, los resultados obtenidos reflejaban como afectan positivamente sobre la productividad. Koleda (2004) muestra que el efecto de los requerimientos de patente novedosa sobre el crecimiento del PIB puede exhibir una forma de $U$ invertida, implicando que una política de protección de propiedad intelectual mas fuerte puede frenar el crecimiento económico de una economía, y demostrando que existe un nivel optimo de requerimientos que maximiza el crecimiento económico. Otros fenómenos que se han observado han sido que los efectos de la I+D sobre las patentes no son constantes, sino que dependen de la calidad de la patente.Asimismo, el efecto sobre la productividad que obtienen las empresas depende en gran medida del número de patentes que esa empresa ya ha realizado en el pasado. Por lo que cuanto antes inicien las empresas el proceso innovador, más patentes obtendrán y mayor será su capacidad de absorción de nuevas ideas, obteniendo mayores resultados. La innovación es la principal fuente de crecimiento y el número de patentes está muy relacionado con la capacidad innovadora y productiva del país. La principal ventaja que proporcionan las patentes es la difusión generalizada de la innovación en la descripción de la patente, lo que ayuda a otras empresas a beneficiarse de esa innovación y aumentar los efectos colaterales sobre la productividad de otras empresas y sobre la economía en general (Blazsek y Escribano, 2010).

\section{Innovaciones de producto}

La variable innovación de producto es importante en el análisis de la productividad, así lo reflejan por ejemplo Huergo y Moreno (2004) en su estudio donde constatan que las empresas cuyas actividades tecnológicas han dado lugar a innovaciones de proceso y/o producto presenta un crecimiento de la productividad global muy superior al resto de empresas.
En un estudio realizado por Calvo González (2000) a las empresas manufactureras españolas, pone de manifiesto que el $30 \%$ de su cifra de negocios se debe a la introducción de nuevos productos procedentes de la innovación que se ha llevado a cabo en ellas. En este sentido Vega Jurado (2008) obtiene el mismo resultado, el mayor impacto sobre la cifra de negocios la presentan los nuevos productos. Cabe destacar que la mayor parte de las innovaciones de producto fueron catalogadas como de un alto grado de novedad (productos nuevos para el mercado). Además también obtiene que las empresas manufactureras españolas, en su conjunto, tienden a innovar más en producto que en proceso.

La creación de nuevos productos surge a partir de las nuevas necesidades que surgen en la sociedad. En este sentido el impacto de la I+D en la productividad se debe a la aplicación de los nuevos conocimientos generados en nuevos productos y nuevos procesos de producción (Czarnitzki y otros, 2007).

\section{Innovaciones de proceso}

La literatura encuentra relaciones entre la innovación de procesos y el crecimiento de la productividad. Smolny (1998), supone que las innovaciones de proceso reducen los costes de producción mediante el aumento de la productividad del trabajo y/o capital. Ericson y Pakes (1992) establecen un modelo de aprendizaje activo, la relación entre las actividades I+D y el crecimiento de la productividad pasa por la consecución y aplicación de las innovaciones de proceso.

En estudios más recientes sigue confirmándose la misma tendencia. Rochina y otros (2008) en sus resultados obtienen que las empresas que implementan procesos de innovación disfrutan de una mayor productividad que las que no lo hacen. Este aumento de la productividad también depende del tamaño de la empresa, las empresas pequeñas ven aumentada su productividad nada más implementar el nuevo proceso, incluso en el mismo año de su instauración. Por el contrario, las grandes firmas pueden tardar hasta dos años en ver los efectos positivos de la implantación del nuevo proceso sobre el aumento de su productividad.

La innovación del procesos también se presenta como un resultado para el no estancamiento de la productividad (Huego y otros, 2004). Las empresas con relativamente poco tiempo de vida tienen a mostrar mayores tasas de crecimiento de la productividad, que con el tiempo, tienen a converger en promedios de tasas de crecimiento comunes. La innovación de procesos influye claramente en el crecimiento de la productividad adicional en cualquier momento del ciclo de vida en el que se encuentre la empresa. 


\section{Organización interna de la innovación}

Con organización interna de la innovación nos referimos a los mecanismos que utiliza la empresa internamente para alcanzar el objetivo de innovar. En este sentido, alguno de ellos pueden ser: mantener una dirección o comité de tecnología, contar con un plan de actividades de innovación, elaborar indicadores de resultados de la innovación obtenida o evaluar las tecnologías alternativas que existen para la empresa. El objetivo no es solo determinar si las empresas disponen de dichas actividades, sino estudiar si influyen positivamente sobre el aumento de su productividad.

En la actualidad, uno de los factores determinantes de la innovación que se considera que más puede estimular la innovación es la cultura organizativa. Esto se debe a que al influir en el comportamiento de los empleados, puede lograr que acepten la innovación como un valor fundamental en la organización y se comprometan con ella (Hartmann, 2006). Las empresas innovadoras necesitan en su organización interna un alto nivel de educación y cualificación del personal técnico, así como experiencia del mismo en el desarrollo de su trabajo. La experiencia acumulada y la cualificación del capital humano de la empresa son dos factores clave para mejorar la productividad de la misma (Baldwin y Johnson, 1996). Milgrom y Roberts (1995) presentan un modelo global de empresa en el que las decisiones relativas a la organización del trabajo, a la gestión del personal, a la subcontratación y a la elección de una tecnología, son complementarias. Muestran que un sistema de producción eficaz es un todo, un conjunto de elementos complementarios.

El tiempo para difundir y adaptar la tecnología se reduce con la adecuada formación de los trabajadores y con cambios en la organización interna de las empresas destinados a un uso más eficiente de la nueva maquinaria (Camacho López y Moreno Galbis, 2006). Los estudios referidos a la importancia de los cambios organizativos promovidos por la adopción de las tecnologías de la información y la comunicación (TIC) son más recientes. Askenazy y Gianella (2000) y Bresnahan y otros (2002) muestran empíricamente la importancia de acompañar toda adopción tecnológica en una empresa con un cambio en la organización del trabajo si se desea obtener un impacto positivo en la productividad.

\section{Externalización de la I+D}

Lockshin y otros (2007) obtienen evidencias econométricas a nivel micro de que existen ganancias en la productividad para aquellas empresas que combinan las estrategias de $I+D$ interna y externa.Asimismo identifican un efecto positivo de la I+D externa cuando el nivel de los gastos de I+D interno es el suficiente. En esta línea Vargas y otros (2007) obtuvieron como resultado que invertir en capital tecnológico tanto interna como externamente tiene efectos positivos sobre la productividad. Pero es importante tener en consideración el tipo de estrategia que se sigue en función del tipo de inversión que se está dispuesto a realizar. Las empresas que optan por invertir pequeñas cantidades de recursos en las actividades tecnológicas tendrán mejores resultados si utilizan el desarrollo externo. Por el contrario si la empresa opta por invertir grandes cantidades de recursos en las actividades de innovación obtendrá mejores resultados si decide desarrollar actividades internas.

Existen otros estudios que ponen en tela de juicio la efectividad de la externalización de la I+D como estrategia de innovación empresarial.Vega-Jurado y otros (2008) concluyen que la I+D externa aplicada de manera individual no ejerce ningún efecto significativo sobre el desempeño innovador de las empresas manufactureras españolas. Las empresas fallan en la asimilación e integración de la I+D adquirida externamente. En contra a lo anterior, las actividades de I+D internas como la compra de maquinaria y equipos se muestra como un determinante importante del desempeño innovador de las empresas.

Zahra y Nielsen (2002) determinan que las principales deficiencias de la I+D externa se derivan del hecho de que las tecnologías adquiridas en el mercado también están a disposición de otras empresas de la competencia, por lo que no pueden ofrecer una ventaja competitiva sostenible. Además, los activos adquiridos externamente no siempre encajan con el saldo de los activos y los procesos de la empresa, requiere de nuevas habilidades y conocimientos más allá de sus límites.

\section{Financiación pública de la I+D}

Numerosos estudios tratan de estimar cuál es el impacto de estas ayudas sobre las empresas y entre otros factores como influyen sobre la productividad. Huergo y Moreno (2004) en este sentido encuentran la financiación pública como un instrumento eficaz para incentivar la intensidad inversora en I+D. En su estudio determinan que las ayudas públicas a la $I+D$ y la inversión privada no son sustitutivas, sino que aparecen como actividades complementarias. La reducida proporción de empresas industriales pequeñas que recibe subvenciones en España (Cotec, 2000) demuestra que el sistema existente aún no es lo suficientemente eficaz para incentivarlas, apuntando la necesidad de mejorarlo para facilitar el acceso de las empresas de menor tamaño.

A pesar del sólido fundamento teórico y el carácter generalizado de este tipo de ayudas, la evidencia sobre su impacto en las acciones de la empresa es relativamente modesta y polémica. Las investigaciones realizadas por Jaumandreu (2004) se han centrado en tratar de determinar si las sub- 
venciones estimulan los gastos de I+D, en el sentido de que las empresas emprenden proyectos de investigación que no hubieran llevado a cabo en otro caso, y si los fondos públicos "desplazan" o no gasto privado de las empresas. Finalmente llega a la conclusión de que las subvenciones son potencialmente efectivas en la inducción de las empresas a emprender actividades de I+D. Las empresas de mayor tamaño con financiaciones modestas serían inducidas a innovar al igual que las de menor tamaño, pero estas con una subvención de mayor capital. En un estudio posterior Jaumandreu (2009) concluye que tanto las deducciones fiscales y subvenciones parecen haber jugado un papel, aunque modesto, en la estimulación de las inversiones en I + D. Las razones de los débiles resultados parecen estar en su coste de aplicación en el caso de las deducciones fiscales, y su aplicación conservadora en el caso de las subvenciones. Los estudios señalan que hay un papel para el incremento de los recursos dedicados a este tipo de políticas, así como una aplicación más eficaz de los incentivos en cuestión.

\section{Colaboración exterior de la I+D}

La colaboración exterior de la innovación implica una participación activa en proyectos de innovación conjunta con otras organizaciones, bien sean otras empresas innovadoras, clientes, proveedores, universidades o centros tecnológicos entre otros. La colaboración se diferencia de los otros flujos de difusión de información en que todos los participantes toman una parte activa en el trabajo. La cooperación en innovación permite a las empresas acceder a unos conocimientos y a unas técnicas que serian incapaces de utilizar por sí mismas. Además aporta un importante potencial de sinergias, ya que permite el aprendizaje mutuo entre los socios (OCDE Eurostat, 2005).

La innovación depende no sólo de factores internos a las empresas, sino también de los procesos interactivos que involucran relaciones entre ellas y otros agentes externos. Para estimular la innovación, las empresas podrán recabar información de los clientes, proveedores, universidades, instituciones de investigación, autoridades públicas, consultores, prensa o ferias comerciales entre otros (Santamaría y otros, 2009; Hagedoorn, 2002). Por lo tanto, la capacidad de una empresa para adquirir, utilizar y desarrollar valiosos recursos y capacidades a menudo depende de la adquisición y absorción de conocimiento externo a través de colaboraciones con el exterior (Teece y otros, 1997).

Vega-Jurado y otros (2009) en su estudio afirman que las empresas no son autosuficientes sobre los recursos tecnológicos y que necesitan combinar sus capacidades con las capacidades que les pueden aportar a través de la colaboración otras empresas e instituciones. Las actividades de I+D promueven el uso de las fuentes externas de cono- cimiento e incrementa la eficiencia del desarrollo de nuevos productos y procesos. La adquisición de conocimiento externo proporciona ideas y recursos para llevar a cabo innovaciones y que estas se desarrollen con existo en el mayor de los casos.

En la misma línea Trigo (20II) destaca que las colaboraciones y contactos informales juegan un papel importante $y$ decisivo en la transmisión de la información y en la creación de conocimiento en las empresas, favorecen a aumentar a su vez la productividad obtenida por estás. Además señala la proximidad geográfica como un factor relevante entre los contactos a la hora de colaborar.

La capacidad de una empresa para adquirir, utilizar y desarrollar valiosos recursos y capacidades a menudo depende de la adquisición y absorción de conocimiento externo a través de colaboraciones con el exterior (Teece y otros, 1997). Vega-Jurado y otros (2009) afirman que las empresas no son autosuficientes sobre los recursos tecnológicos y que necesitan combinar sus capacidades con las de otras empresas e instituciones con las que colaboran.

\section{Datos, modelo y metodología}

Analizamos información homogénea de 1872 empresas de 20 sectores manufactureros en 2010. La fuente de información es la Encuesta sobre Estrategias Empresariales (ESEE) realizada por el Ministerio de Industria de España. El criterio de selección muestral es muestreo estratificado aleatorio por sectores y tamaños a partir de una muestra representativa de la población.

\begin{tabular}{|c|l|c|}
\hline Número & Factores de innovación & Signo \\
\hline I & $\begin{array}{l}\text { Realización o contratación de ac- } \\
\text { tividades I+D }\end{array}$ & Positivo \\
\hline 2 & Gasto en I+D & Positivo \\
\hline 3 & Número de patentes & Positivo \\
\hline 4 & Innovaciones de producto & Positivo \\
\hline 5 & Innovaciones de proceso & Positivo \\
\hline 6 & $\begin{array}{l}\text { Organización interna de la inno- } \\
\text { vación }\end{array}$ & Positivo \\
\hline 7 & $\begin{array}{l}\text { Externalización de actividades } \\
\text { I+D }\end{array}$ & Negativo \\
\hline 8 & Financiación pública de la I+D & Positivo \\
\hline 9 & Colaboración exterior de la I+D & Positivo \\
\hline
\end{tabular}

Tabla I. Hipótesis. Efecto esperado sobre la productividad de los factores de innovación 
Para estudiar los factores de innovación determinantes de la productividad de la empresa industrial española, hemos incluido dichos factores en la función de producción, cuya expresión es la siguiente:

$\operatorname{Ln}(\mathrm{VAi})=\alpha+\beta 1 \ln (\mathrm{GLABi})+\beta 2 \ln (\mathrm{CAPi})+\beta 3 \ln (\mathrm{NLABi})$

$+\beta 4 \mathrm{GIDi}+\beta 5 \mathrm{AIDTi}+\beta 6 \mathrm{AIDEi}+\beta 7 \mathrm{NPATi}+\beta 8 \mathrm{INPDi}$ $+\beta 9 \mathrm{INPCi}+\beta 10 \mathrm{FPUBi}+\beta 11 \mathrm{OINTi}+\beta 12 \mathrm{CEXTi}+$ $\Sigma \beta S S E C T i, s+\varepsilon i \quad(1)$

Gracias precisamente a su forma logarítmica puede estimarse como una regresión lineal múltiple.A continuación se detalla el contenido de las variables del modelo:

$\alpha$ es el término que representa el nivel tecnológico.

VA (Valor Añadido) es el output medido como el valor añadido, se obtiene sumando ventas, variación de existencias de ventas, otros ingresos de gestión corriente, variación de existencias de compras y restando las compras, así como los servicios exteriores.

GLAB (Gasto Laboral) es el gasto del input del trabajo determinado mediante el gasto laboral.

CAP (Capital) es el servicio input capital definido como el stock de capital, se mide como el inmovilizado material.

NLAB (Número de trabajadores) es el servicio input trabajo expresado por medio del número de trabajadores.

GID (Gastos de I+D) es el cociente de los gastos de I+D entre el stock de capital.

AIDT (Actividades de I+D internas y externas) indica si las actividades de $I+D$ se realizan internamente o externamente. La variable toma valor cero si no se realizan, toma valor uno si se realizan internamente o externamente y toma valor dos si se realizan internamente y externamente.

AIDE (Actividades de I+D externas) indica si las actividades de I+D se realizan externamente. Toma valor uno si se realizan externamente y cero si no se realizan externamente. NPAT (Número de Patentes) es el número de patentes obtenidas, indica en cierta medida el resultado obtenido por la I+D.

INPD (Innovaciones de Producto) indica cuántas de las siguientes innovaciones se han obtenido: a) Incorpora nuevos materiales, b) Incorpora nuevos componentes o productos intermedios, c) Incorpora nuevo diseño y presentación, d) El producto cumple nuevas funciones. Esta variable toma valores entre cero y cuatro. Por ejemplo: si sólo incorpora nuevos materiales toma valor uno, si incorpora nuevos materiales $y$ el producto cumple nuevas funciones toma valor dos, y así sucesivamente. Si no obtiene ninguna toma valor de cero.

INPC (Innovaciones de Proceso) indica cuántas de las siguientes innovaciones se han realizado: a) Introducción de nueva maquinaria, b) Introducción de nuevos programas informáticos, c) Introducción de nuevos métodos de organización de producción. Toma valores entre cero y tres. De este modo, si sólo se introduce una nueva máquina toma valor uno, si introduce una nueva máquina y unos nuevos programas informáticos toma valor dos, $y$ así sucesivamente. Si no obtiene ninguna toma valor cero.

FPUB (Financiación Pública) es el cociente de la financiación pública para la I+D entre la variable GID (cociente de los gastos de I+D entre el stock de capital).

OINT (Organización Interna) indica cuántos de los siguientes mecanismos dispuso la empresa o de las siguientes acciones realizó, en relación con la organización interna de la innovación: a) Mantener una dirección o comité de tecnología o I+D, b) Contar con un plan de actividades de innovación, c) Elaborar indicadores de resultado de la innovación, d) Evaluar tecnologías alternativas para la empresa. Toma valores entre cero y cuatro. Por ejemplo: si sólo elabora indicadores de resultado de la innovación toma valor uno, si elabora indicadores de resultado de la innovación y evalúa tecnologías alternativas para la empresa toma valor dos, y así sucesivamente. Si no obtiene ninguna toma valor cero.

CEXT (Colaboración Exterior) indica cuántos de los siguientes mecanismos dispuso la empresa 0 de las siguientes acciones realizó, en cuanto a la colaboración exterior de la innovación: a) Colaborar con Universidades y/o centros tecnológicos, b) Colaborar tecnológicamente con clientes, c) Colaborar tecnológicamente con proveedores, d) Colaborar tecnológicamente con competidores, e) Mantener acuerdos de cooperación tecnológica (Joint Ventures), f) Participar en empresas que desarrollan innovación tecnológica, g) Incorporar ingenieros y/o licenciados de graduación reciente, $h$ ) Reclutar personal con experiencia empresarial en I+D, i) Financiar la innovación con créditos subvencionados, j) Participar en programas de investigación de UE. Toma valores entre cero y diez. Por ejemplo: si sólo colabora tecnológicamente con clientes toma valor uno, si colabora tecnológicamente con clientes y con proveedores toma valor dos, y así sucesivamente. Si no obtiene ninguna toma valor cero.

$\Sigma \beta S S E C T i, s$ (Sectores manufactureros) en esta suma SECTi,s vale uno si la empresa i pertenece al sector s. En otro caso toma el valor cero. De este modo, el coeficiente $\beta S$ representa el efecto asociado al sector $\mathrm{s}$.

$\varepsilon i$ (Error) es el término de error. 


\section{Resultados y discusión}

Todas las estimaciones fueron obtenidas con Stata 9.0 y son proporcionados en la tabla 2 junto con los estadísticos descriptivos de las variables de la expresión (I). La tabla 3 proporciona la matriz de correlación de dichas variables. Tanto los estadísticos descriptivos como la matriz de correlación facilita la interpretación de los resultados de regresión. El coeficiente de correlación (R2) del modelo es de 0,9492 , un valor muy próximo a uno, por lo que la bondad del ajuste es elevada.
Los resultados de estimación muestran que los coeficientes que presentan el capital (CAP) y el trabajo (NLAB), son de 0,1787 y 0,8382 respectivamente. La suma de ambos resulta 1,0169 por lo que hay rendimientos a escala levemente crecientes. Esto quiere decir que la productividad obtenida aumenta en mayor proporción a lo que lo hacen los inputs. Tal y como pronosticábamos, la realización de actividades I+D influye positivamente sobre la productividad, quedando confirmada la hipótesis I. Mientras que la externalización de actividades de I+D no es significativa por lo que no se puede

\begin{tabular}{|c|c|c|c|c|c|c|}
\hline Variables & Media & Desviación típica & Mínimo & Máximo & $\begin{array}{c}\text { Coeficiente esti- } \\
\text { mado }\end{array}$ & p-valor \\
\hline $\ln ($ VA) & 14,6981 & 1,7546 & 9,1410 & 21,9702 & & \\
\hline$\alpha$ & & & & & 0,7900 & $0,010\left(^{*}\right)$ \\
\hline $\ln ($ GLAB) & 10,3728 & 0,3894 & 8,9028 & 12,6420 & 0,7467 & $0,000\left(^{*}\right)$ \\
\hline $\ln (\mathbf{C A P})$ & 15,5993 & 1,9667 & 10,5811 & 22,1783 & 0,1787 & $0,000\left(^{*}\right)$ \\
\hline $\ln (\mathbf{N L A B})$ & 4,0579 & 1,4049 & 0,0000 & 9,2680 & 0,8382 & $0,000\left(^{*}\right)$ \\
\hline GID & 0,0150 & 0,0577 & $5,00 \mathrm{E}-07$ & 1,2248 & 0,0487 & 0,794 \\
\hline AIDT & 0,5534 & 0,7957 & 0 & 2 & 0,0831 & $0,012\left(^{* *}\right)$ \\
\hline AIDE & 0,2387 & 0,4264 & 0 & 1 & $-0,0236$ & 0,622 \\
\hline NPAT & 0,3370 & 6,7862 & 0 & 288 & 0,0000 & 0,986 \\
\hline INPD & 0,5416 & 1,0661 & 0 & 4 & 0,0024 & 0,814 \\
\hline INPC & 0,6362 & 0,9801 & 0 & 3 & 0,0541 & $0,000\left(^{*}\right)$ \\
\hline FPUB & 129042,3 & 3823876 & 0 & $1,40 \mathrm{E}+08$ & $3,44 \mathrm{E}-10$ & 0,887 \\
\hline OINT & 0,8600 & 1,3652 & 0 & 4 & $-0,0177$ & 0,135 \\
\hline CEXT & 1,0064 & 1,6988 & 0 & 9 & $-0,0059$ & 0,519 \\
\hline
\end{tabular}

Nota: Los valores con $(*),\left({ }^{*}\right)$ y $(* * *)$ indica un nivel de significación del $1 \%, 5 \%$ y $10 \%$ respectivamente Tabla 2. Estadísticos descriptivos y resultados de regresión

\begin{tabular}{|c|c|c|c|c|c|c|c|c|c|c|c|c|c|c|}
\hline \multicolumn{2}{|c|}{ Variables } & $\mathrm{I}$ & 2 & 3 & 4 & 5 & 6 & 7 & 8 & 9 & 10 & $\mathrm{II}$ & 12 & 13 \\
\hline $\mathrm{I}$ & $\operatorname{In}(\mathrm{VA})$ & $\mathrm{I}$ & & & & & & & & & & & & \\
\hline 2 & $\ln (\mathrm{GLAB})$ & 0,60 & $\mathrm{I}$ & & & & & & & & & & & \\
\hline 3 & $\operatorname{In}(\mathrm{CAP})$ & 0,92 & 0,58 & $\mathrm{I}$ & & & & & & & & & & \\
\hline 4 & $\operatorname{In}(\mathrm{NLAB})$ & 0,95 & 0,45 & 0,89 & $\mathrm{I}$ & & & & & & & & & \\
\hline 5 & $\mathrm{GID}$ & $0, \mathrm{I}$ & 0,14 & 0,04 & $0, \mathrm{I}$ & $\mathrm{I}$ & & & & & & & & \\
\hline 6 & $\mathrm{AIDT}$ & 0,53 & 0,34 & 0,48 & $0,5 \mathrm{I}$ & 0,36 & $\mathrm{I}$ & & & & & & & \\
\hline 7 & $\mathrm{AIDE}$ & 0,44 & 0,27 & 0,40 & 0,43 & 0,29 & 0,88 & $\mathrm{I}$ & & & & & & \\
\hline 8 & $\mathrm{NPAT}$ & 0,05 & 0,02 & 0,03 & 0,05 & 0,03 & 0,02 & $0,0 \mathrm{I}$ & $\mathrm{I}$ & & & & & \\
\hline 9 & $\mathrm{INPD}$ & 0,32 & 0,18 & 0,27 & 0,32 & 0,18 & 0,44 & 0,35 & $0,0 \mathrm{I}$ & $\mathrm{I}$ & & & & \\
\hline $\mathrm{I} 0$ & $\mathrm{INPC}$ & 0,36 & 0,17 & 0,32 & 0,35 & 0,12 & $0,4 \mathrm{I}$ & 0,34 & 0,07 & 0,46 & $\mathrm{I}$ & & & \\
\hline $\mathrm{II}$ & $\mathrm{FPUB}$ & $0,0 \mathrm{I}$ & $0,0 \mathrm{I}$ & $0,0 \mathrm{I}$ & $0,0 \mathrm{I}$ & $-0,0 \mathrm{I}$ & $0,0 \mathrm{I}$ & $-0,02$ & $-0,0 \mathrm{I}$ & $0,0 \mathrm{I}$ & $-0,02$ & $\mathrm{I}$ & & \\
\hline $\mathrm{I} 2$ & $\mathrm{OINT}$ & 0,52 & 0,35 & 0,48 & 0,50 & 0,29 & 0,74 & 0,57 & 0,03 & 0,49 & 0,46 & $-0,0 \mathrm{I}$ & $\mathrm{I}$ & \\
\hline $\mathrm{I} 3$ & $\mathrm{CEEXT}$ & 0,53 & 0,36 & 0,49 & 0,53 & 0,38 & 0,73 & $0,6 \mathrm{I}$ & 0,02 & 0,43 & 0,42 & $-0,0 \mathrm{I}$ & 0,73 & $\mathrm{I}$ \\
\hline
\end{tabular}

Tabla 3. Matriz de correlación

ISSN: 07 I8-2724. (http://www.jotmi.org)

Journal of Technology Management \& Innovation (c) Universidad Alberto Hurtado, Facultad de Economía y Negocios. 
aceptar la hipótesis 7. Así, a partir de estos dos resultados de estimación, se interpreta que las actividades de I+D internalizadas favorecen en mayor medida la productividad, pudiendo llegar a afectar negativamente cuando se externalizan. Este efecto positivo de las actividades de I+D internalizadas mayor que en las actividades de I+D externalizadas está acorde con que se esperaba y con la literatura.

Sin embargo, los gastos de I+D no son significativos, lo que impide aceptar la hipótesis 2. Esta inversión está incluida en el input de capital, estimado como un stock. Como este coeficiente puede ser cero, este tipo de capital tiene la misma elasticidad del output del resto de los activos. Es probable que la explicación de esto esté en la medida de los gastos de I+D en la ESEE que los contabiliza solamente cuando han sido incluidos como activos fijos. Podemos interpretar que no es tan importante la cantidad invertida en I+D, sino su correcta realización.

Las patentes no tienen un efecto significativo en la productividad, por lo que no es posible aceptar la hipótesis 3. A pesar de ello, han sido incluidas en el modelo puesto que la posesión de patentes por parte de la empresa, implica ésta explote esta ventaja, se dificulta su apropiación, se favorezca la privacidad y que no se tenga interés en aprovechar nuevas mejoras.

Las innovaciones de proceso son significativas, con grado de significación $1 \%$, positiva y con elasticidad del $0,054 \mathrm{I}$, confirmando la hipótesis 5 . Lo que implica que la productividad aumentará en un $5,4 \%$ en cada innovación de proceso que se realice en la empresa. Son datos muy positivos pues un aumento de la productividad en dicho porcentaje supone un estímulo para la innovación. Así, las innovaciones de proceso favorecen la generación de conocimiento tecnológico y por tanto de la productividad.

No es posible confirmar la hipótesis 6 dado que no resulta significativa la relación entre la organización interna de la innovación y la productividad. Una posible explicación para este resultado, puede ser que las empresas manufactureras españolas tengan múltiples y variadas formas de organizar internamente la innovación, conduciendo a que sea irrelevante dicho factor sobre la productividad.

Tanto la innovaciones en el producto, la financiación pública de la I+D como la colaboración exterior de la innovación tampoco tienen efecto significativos sobre la productividad, lo que nos lleva a no poder aceptar la hipótesis 4, 8 ni la 9 . Este resultado pueden conducir a decir que el efecto de la innovación sobre la productividad viene determinado por otros factores diferentes de las innovaciones en el producto, la financiación pública de la I+D y de la colaboración exterior de la innovación.

\section{Conclusiones}

La innovación es una fuente de productividad y nuestros resultados así lo recogen en función de los estudios previos. Para ello, hemos estudiado datos a 1872 empresas de 20 industrias en 2010, mediante la estimación de un modelo de función de producción que explica los factores de innovación determinantes del valor añadido.

Dicha función de producción incluye el efecto de los siguientes factores de innovación sobre la productividad en las empresas manufactureras españolas: actividades $I+D$, gastos I+D, patentes, innovaciones de producto, innovaciones de proceso, organización interna de la innovación, externalización de las actividades de I+D, financiación pública de los gastos de I+D y colaboración exterior de la innovación.

Nuestros hallazgos recogen que las actividades de I+D favorecen la productividad de la empresa manufacturera española. Las actividades de I+D internas tienen el efecto más positivo, mientras que las externalizadas pueden llegar a ser negativas. Esto explica que la decisión de externalizar el I+D para innovaciones menores que requieres inversiones en activos no específicos; siendo su contribución sobre la productividad menor.

Además, las innovaciones en el proceso permiten tener mejores herramientas para llevar a cabo la actividad.Así, confirmamos que dicho proceso productivo innovador favorece la aplicación y producción de conocimiento necesario para la mejora de la productividad.

Las contribuciones de este trabajo abren líneas de investigación futuras, tales como el estudio de las relaciones verticales entre las empresas (con proveedores y clientes). 


\section{Agradecimientos}

Los autores agradecen los fondos recibidos por MINE$\mathrm{CO}$ a través del proyecto de investigación ECO201236290-C03-0I.

\section{Referencias}

ASKENAZT, P., Gianella, C. (2000). Le Paradoxe de Productivité: les Changements Organisationnels, facteur complémentaire à l'informatisation. Economie et Statistique, 9/10, 219-242. doi:http://dx.doi.org//0.3406/estat.2000.7486

BALDWIN, J., Johnson, J. (1996). Business Strategies in More- and Less- Innovative Firms in Canada. Research Policy, 25(5), 785-804. doi:http://dx.doi.org/10.1016/00487333(95)00875-6

BARGE-GIL, A., López, A. (20II). Realización de I+D y su composición en la empresa manufacturera española, análisis de los determinantes diferenciados de la investigación y el desarrollo. Economía industrial, 382, 25-34.

BENITO, P. (200I). R\&D productivity and spillovers at the firm level: evidence from Spanish panel data. Investigaciones Económicas, 25, 289-3। 3.

BLAZSEK, S., Escribano, A. (20I0). Knowledge spillovers in US patents: A dynamic patent intensity model with secret common innovation factors. Journal of Econometrics, I59(I), 14-32. doi:http://dx.doi.org//0.1016/j.jeconom.2010.04.004

BRESNAHAN, T., Brynjolfsson, E., Hitt, L.M. (2002). Information Technology, Workplace organization, and the Demand for skilled Labor: Firm-Level Evidence. The Quarterly Journal of Economics, II7(I), 339-376. doi:http://dx.doi. org/10.1 I62/003355302753399526

CALVO-GONZÁLEZ, J. (2000). Una caracterización de la innovación tecnológica en los sectores manufactureros españoles. Economía industrial, 33I, I39-I50.

CAMACHO-LÓPEZ, C., Moreno-Galbis, E. (2006). Difusión tecnológica, productividad y cambio organizativo. Cuadernos económicos de ICE, 72, 255-280.

COTEC (2000). Relación para la innovación de las empresas con las administraciones. Informes sobre el sistema español de innovación.

CRÈPON, B., Duguet, E., Mairesse, J. (1998). Research, Innovation and Productivity: an Econometric Analysis at the Firm Level. Economics of Innovation and New Technology, 7(2), I I5-I58. doi:http://dx.doi.org//0.1080//043859980000003 |

CZARNITZKI, D., Niall O'Byrnes (2007). The impact of R\&D on productivity. FORFAS (Ed.), Perspectives on Irish Productivity, Dublin, 315-329. 
DORASZELSKI, U., Jaumandreu, J. (2007). R\&D and productivity: estimating production functions when productivity is endogenous. Harvard Institute of Economic Research, Discussion Paper, 2147, I-55. doi:http://dx.doi.org//0.2/39/ ssrn. 1080306

ERICSON, R., Pakes, A. (1992). An alternative theory of firm and industry dynamics. Cowles Foundation Discussion Papers, Yale University, I04I.

FLUVIÁ-FONT, M. (1990). Capital tecnológico y externalidades: un análisis de panel. Investigaciones Económicas, 15, suplemento, 167-172.

GRANDÓN, V., Rodríguez-Romero, L. (1991). Capital tecnológico e incremento de productividades en la industria española, 1975-198I. Investigaciones Económicas, 15, suplemento, 19-24.

GRIFFITH, R., Redding, S.,Van Reenen, J. (2004). Mapping the two faces of R\&D: productivity growth in a panel of OECD industries. The Review of Economics and Statistics, 86(4), 883-895. doi:http://dx.doi.org/ I 0. I | 62/0034653043 | 25 I 94

GRILICHES, Z. (1979). Issues in assessing the contribution of research and development to productivity growth. The Bell Journal of Economics, 10(I), 92-I16. doi:http://dx.doi. org/ $0.2307 / 300332$ |

GRILICHES, Z., Mairesse, J. (1984). Productivity and R+D at the Firm Level. Zvi Griliches, ed. University of Chicago Press, 339-374. doi:http://dx.doi.org// 0.3386/w0826

GU,W., Tang, J. (2004). The link between innovation and productivity in Canadian manufacturing industries. Economics of Innovation and New Technology, I3(7), 67I-686. doi:http:// dx.doi.org/I0.1080/I0438904I000I686806

HAGEDOORN, J. (2002). Inter-Firm R\&D Partnerships: An Overview of Major Trends and Patterns since 1960. Research Policy, 3 I (4), 477-492. doi:http://dx.doi.org//0.1016/ s0048-7333(0I)00I 20-2

HARTMANN, A. (2006). The role of organizational culture in motivating innovative behaviour in construction firms. Construction Innovation, 6, 159-172. doi:http://dx.doi. org//0.1108/I47/41706/07/07/2

HUERGO, E., Moreno, L. (2004). La innovación y el crecimiento de la productividad en España. Ekonomiaz: Revista vasca de economía, 56, 208-23I.
JAUMANDREU,J (2004). Cuatro estudios microeconómicos sobre temas de innovación. Estudio 26, COTEC, Fundación para la innovación tecnológica.

JAUMANDREU, J. (2009). What explains the evolution of productivity and competitiveness? The innovation Link. IESE Business School University of Navarra, Working Paper, 804, I-37. doi:http://dx.doi.org//0.2139/ssrn. 1482990

KOLEDA, G. (2004). Patents Novelty Requirement and Endogenous Growth. Revue d'économie politique, II4, 20I221 .

LOCKSHIN, B., Belderbos, R., Carree, M. (2007). The productivity effects of internal and external R\&D: Evidence from a dynamic panel data model. Oxford bulletin of Economics and Statistics, 70(3), 399-413. doi:http://dx.doi.org//0.1 I I I/ j. $1468-0084.2008 .00503 . x$

LÓPEZ-PUEYO, C., Sanaú-Villarroya, J. (1998). Capital tecnológico y productividad en la industria española. Comunicación presentada en el I Encuentro de Economía Aplicada, Barcelona.

MATÉ GARCÍA, J. J., Rodríguez Fernández, J. M. (2002). Crecimiento de la productividad e innovación en I+D: Un análisis empírico de las empresas manufactureras españolas. Economía Industrial, 1347, 99-I I0.

MILGROM, P., Roberts, J. (1995). Complementarities and Fit Strategy, Structure and Organizational Change in Manufacturing. Journal of Accounting and Economics, I9(I), I79-208. doi:http://dx.doi.org/I0.1016/0165-4I0I(94)00382-F

OBSERVATORIO DE LA SOSTENIBILIDAD EN ESPAÑA (20II). Informe anual sobre Sostenibilidad en España en 20I I: Inversión en I+D en relación al PIB. Temática: Desarrollo socioeconómico, modelos de producción y consumo.

OCDE, Eurostat (2005). Manual de Oslo: Guía para la recogida e interpretación de datos sobre innovación. $3^{a}$ edición, Tragsa.

ROCHINA-BARRACHINA, M., Mañez, J., Sanchis-Llopos, J. (2008). Process innovations and firm productivity growth. Small Business Economics, 34, 147-166. doi:http://dx.doi. org/| 0.1007/s | | |87-008-9|| |0-5

ROSELL-MARTíNEZ, J., Sánchez-Sellero, P. (201 I). Foreign direct investment and technical progress in Spanish manufacturing. Applied Economics, 44(19), 2473-2489. doi:http:// dx.doi.org/I0.1080/00036846.20II.564I55 
SANTAMARIA, L., Nieto, M., Barge-Gil, A. (2009). Beyond formal R\&D: Taking advantage of other sources of innovation in low- and medium-technology industries. Research Policy, 38(3), 507-5/7. doi:http://dx.doi.org//0.1016/j.respol.2008.10.004

SMOLNY, W. (1998). Innovations, prices and employment: A theoretical model and an empirical application for West German manufacturing firms. The Journal of Industrial Economics, 46(3), 359-38I. doi:http://dx.doi.org/I0.1 I I I/I4676451.00076

TEECE, D., Pisano, G., Shuen, A. ( 1997$)$. Dynamic capabilities and strategic management. Strategic Management Journal, 18(7), 509-533. doi:http://dx.doi.org//0.1002/(SICl) 10970266(I 99708) I8:7<509::AID-SMJ882>3.0.CO;2-Z

TRIGO, A. (20I I). Innovación y la importancia de los flujos externos de información: una propuesta para el análisis de los contactos informales. Journal of Technology Management \& Innovation, 6(2), 167-175. doi:http://dx.doi.org//0.4067/ s07|8-272420II0002000II

VARGAS-MONTOYA, P., Salinas-Zárate, R., Guerras-Martín, L. (2007). Does the Technological Sourcing Decision Matter? Evidence from Spanish Panel Data. R\&D Management, 37(2), I6I-I72. doi:http://dx.doi.org//0.1III/j.1467$9310.2007 .00465 . x$

VEGA JURADO,J. (2008). Las estrategias de innovación en la industria manufacturera española: sus determinantes y efectos sobre el desempeño innovador. Tesis doctoral, Universidad Politécnica de Valencia.

VEGA-JURADO, J., Gutiérrez-García, A., Fernández-de-Lucio, l. (2008). ¿Cómo innovan las empresas españolas? Una evidencia empírica. Journal of Technology Management \& Innovation, 3(3), I00-I I I. doi:http://dx.doi.org/I0.4067/s07 I827242008000100010

VEGA-JURADO, J., Gutiérrez-García, A., Fernández-deLucio, I. (2009). Does external knowledge sourcing matter for innovation?: evidence from the Spanish manufacturing industry. Industrial and corporate change, 18(4), 637-670. doi:http://dx.doi.org//0.1093/icc/dtp023

ZAHRA, S., Nielsen,A. (2002). Sources of capabilities, integration and technological commercialization. Strategic Management Journal, 23(5), 377-398. doi:http://dx.doi.org//0.1002/ smj.229 